\title{
MORPHOLOGICAL CHARACTERISTICS OF POTENTIALLY MALIGNANT PULMONARY NODULES IN HIGH-RISK MALE SMOKERS DETECTED IN LUNG CANCER SCREENING TRIAL in Cracow, Poland
}

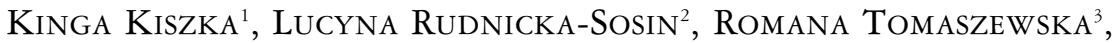 \\ Majgorzata Urbańczyk-Zawadzka ${ }^{1}$, Maciej Krupiński ${ }^{1}$, Patrycja Pikul ${ }^{1}$, Kaja Podsiadeo ${ }^{1}$, \\ Mieczyseaw Pasowicz ${ }^{1}$, Rozemarijn Vliegenthart ${ }^{4}$, Matthijs OudKerk ${ }^{4}$, Tomasz Miszalski-Jamka ${ }^{1}$
}

1Department of Radiology, John Paul II Hospital, Cracow, Poland

2Department of Pathomorphology, John Paul II Hospital, Cracow, Poland

3 Department of Pathomorphology, Jagiellonian University Medical College, Cracow, Poland

${ }^{4}$ Department of Radiology, University of Groningen, The Netherlands

The purpose of this paper was to present morphological characteristics of potentially malignant nodules revealed in a group of male smokers aged 50-74 with a very high risk for developing lung cancer estimated in the study for lung cancer screening in Cracow (Poland). Nine hundred male smokers aged 50 to 74 years were invited to the study and were asked in questionnaires about e.g. smoking exposure history. Exclusion criteria included e.g. positive cancer history and chest computed tomography (CT) examination in the previous year. Based on CT results and characteristics of pulmonary nodules subjects were classified to group A (low risk), group B (indeterminate) and group C (high-risk individuals - required work-up). Final diagnosis was based on pathological results of postoperative material. Thirty-nine males of mean age 63.4 (standard deviation (SD): 6.69 years) revealed 41 potentially malignant pulmonary nodules in baseline screening. In 14 subjects 16 type $C$ pulmonary nodules were histologically proved. Nine nodules were found to be benign lesions, while 7 nodules revealed malignant lung cancer: 5 cases of adenocarcinoma and 2 cases of adenosquamous carcinoma. We determined morphological characteristics of potentially malignant pulmonary nodules in 39 high-risk male smokers and proved lung cancer in 7 subjects.

Key words: lung cancer screening, pulmonary nodules, computer aided diagnosis, adenocarcinoma, pulmonary nodule volumetry.

\section{Introduction}

Pulmonary nodules were common findings in lung parenchyma especially in current and former smokers. Improvement in detection of pulmonary nodules was possible by means of using spiral chest computed tomography (CT) with postprocessing and computer aided diagnosis (CAD) systems [1-5]. There were many attempts in lung cancer screening trials to provide evaluation of solid pulmonary nodules, which could be considered as potentially early stages of lung cancer $[3,5,6]$. However, most of the pulmonary nodules presented benign diseases. Lung cancer is the leading cause of cancer deaths in males, with a mean 5-year survival rate of about 10$15 \%$. Early detection and treatment of lung cancer played an important role in improving treatment and decreasing mortality. The best prognosis of about $70 \% 5$-year survival rate occurred in patients in stage I (T1NOM0). 


\section{Aim of the study}

The purpose of this paper was to present baseline characteristics of potentially malignant nodules revealed in a group of male smokers aged 50-74 with a very high risk for developing lung cancer estimated in the study for lung cancer screening in Cracow (Poland).

\section{Material and methods}

The study was approved by the Polish Ministry of Science and Higher Education and by the ethics board. All participants gave written informed consent.

The participants in the study in Cracow were invited from the population registry and were asked in questionnaires about general health, alcohol consumption, physical exercise, cancer history, family history of lung cancer, body weight and height, level of education and smoking exposure history (years smoked, cigarettes smoked per day, years quit). Exclusion criteria included: current or past renal cancer, melanoma or lung cancer diagnosed less than 5 years ago or lung cancer diagnosed 5 years or more ago but still under treatment, obesity (BMI $\geq 30 \mathrm{~kg} / \mathrm{m}^{2}$ ), NYHA III and IV, chest CT examination in previous year. Additionally, 300 agematched control individuals were enrolled. In all participants low-dose chest CT without contrast media in spiral mode in inspiration was performed using 64 dualsource CT with $0.75-\mathrm{mm}$ slices in cranial-caudal scan direction. $\mathrm{KVp}$ settings were dependent on body weight, and $\mathrm{mAs}$ were adjusted by the machine. Double reading of all images were performed with the LungCare Siemens software package for multi-dimensional image processing and computer viewing. Pulmonary nodule diameter and volume were automatically calculated by means of a 3D (three dimensional) assessment tool, which could be manually corrected by the radiologist using 2D (two dimensional) measurements.

According to NELSON criteria concerning size and morphological features on baseline screening non-calcified solid nodules were classified to three groups. Type A nodules (negative test results) presented benign lesions with morphologically benign features (such as fat or benign calcifications) and/or were smaller than $50 \mathrm{~mm}^{3}$. Type B nodules (indeterminate test results) presented solid nodules of $50-500 \mathrm{~mm}^{3}$ in size. Finally, type $\mathrm{C}$ nodules (positive test results, potentially malignant nodules) were larger than $500 \mathrm{~mm}^{3}$. Based on CT examinations and characteristics of pulmonary nodules, subjects were classified to group A, B or C. Within group A males revealed only type A pulmonary nodules. Males were classified to group B or group C if any pulmonary nodule of type $B$ or type $C$ respectively was observed. Participants from group A with benign nodules required an annual repeat scan. Subjects of group $\mathrm{B}$ with indeterminate nodules required follow-up scanning after 3-4 months to assess growth. Males of group $\mathrm{C}$ were referred to further work-up and histological diagnosis.

There were 39 males who were classified to group $\mathrm{C}$ with a very high risk of lung cancer. All subjects from group $\mathrm{C}$ revealed potentially malignant nodules, which required work-up and histological diagnosis. Of the 39 participants with positive test results, 32 were referred for workup, 3 were not referred (because of the decision of pulmonologists), and 4 participants refused further diagnostics. All 32 participants were diagnosed by means of invasive procedures such as bronchoscopy with EBUS-TBNA (endobronchial ultrasound - transbronchial needle aspiration), in 7 cases PET CT was performed, in 14 males surgical procedures by means of minithoracotomy were performed. Based on intraoperative histopathological examinations, further decisions about broadening the operation range were decided. In case of a malignant nodule, lobectomy with lymphadenectomy was performed. Final diagnosis was based on pathological results of postoperative material.

The analyses were based as the use of $t$ test for normally distributed continuous variables, Mann-Whitney $U$ test, and $\chi^{2}$ test for categorical variables. The prespecified significance level was set at $\alpha=0.05$. All analyses were conducted using the statistical software package STATISTICA v. 10.

\section{Results}

Of the 39 participants of mean age 63.4 (standard deviation (SD): 6.69 years) with positive test results, $33.3 \%(\mathrm{n}=13)$ were younger than 60 years old and $66.7 \%(\mathrm{n}=26)$ were $\geq 60$ years old. $59 \%(\mathrm{n}=23)$ were current smokers and $41 \%(\mathrm{n}=16)$ were former smokers. 33.3\% $(\mathrm{n}=13)$ had positive family cancer history. None of the respondents from group $\mathrm{C}$ revealed exposure to asbestos longer than 10 years. Within 39 males with potentially malignant nodules 4 participants refused further diagnostics, 32 (91.4\% of 35 males) were referred for workup, and 3 ( $8.6 \%$ of 35 males) were not referred (because of the decision of pulmonologists). $33.3 \%(\mathrm{n}=13)$ of subjects revealed exertional cough, $25.6 \%(\mathrm{n}=10)$ permanent cough and $25.6 \%(\mathrm{n}=$ $10)$ morning cough, and $17.9 \%(\mathrm{n}=7)$ spat sputum. None of the participants revealed loss of weight, pneumonia or hemoptysis.

In 39 participants a total of 41 type $C$ pulmonary nodules of median volume $1197 \mathrm{~mm} 3(\bar{x}=3843.8$ $\mathrm{mm}^{3}$ ) were detected. Apart from potentially malignant type $C$ nodules also 156 type $A$ nodules of median volume $17 \mathrm{~mm}^{3}\left(\bar{x}=43.7 \mathrm{~mm}^{3}\right)$ and 54 type $B$ nodules of median volume $99 \mathrm{~mm} 3\left(\bar{x}=140.9 \mathrm{~mm}^{3}\right)$ were observed (Table I). According to location, potentially malignant pulmonary nodules (type $\mathrm{C}$ ) were detected in the right lung in $57.1 \%(n=20)$ and in the upper lobes in $51.4 \%(\mathrm{n}=18)$ (Table II). 
Table I. Volumetry categorized by type of pulmonary nodules within group $\mathrm{C}$ participants. $\mathrm{A}_{\mathrm{C}}, \mathrm{B}_{\mathrm{C}}$ and $\mathrm{C}_{\mathrm{C}}-$ total number of $\mathrm{A}, \mathrm{B}$ and $\mathrm{C}$ nodules within group $\mathrm{C}$ subjects

\begin{tabular}{lccc}
\hline TYPE OF PULMONARY NODULE & \multicolumn{3}{c}{ GROUP $\mathrm{C}$} \\
\cline { 2 - 3 } & $\mathrm{A}_{\mathrm{C}}$ & $\mathbf{B}_{\mathrm{C}}$ & $\mathrm{C}_{\mathrm{C}}$ \\
\hline Total number & 156 & 54 & 41 \\
\hline Summary volumetry of pulmonary nodules $\left[\mathrm{cm}^{3}\right]$ & 6.8 & 7.6 & 157.6 \\
\hline Mean volumetry of single pulmonary nodule $\left[\mathrm{mm}^{3}\right]$ & 43.7 & 140.9 & 3843.8 \\
\hline Median $\left[\mathrm{mm}^{3}\right]$ & 17 & 99 & 1197 \\
\hline
\end{tabular}

All 32 participants were diagnosed by means of invasive procedures such as bronchoscopy with EBUSTBNA (endobronchial ultrasound - transbronchial needle aspiration), in $7(21.9 \%)$ cases PET-CT was performed and in $14(43.6 \%)$ cases surgical procedures by means of minithoracotomy were performed. In $56.3 \%$ ( $\mathrm{n}=18$ ) of cases EBUS-TBNA revealed no changes and in these subjects further control by a pulmonologist was recommended. Based on histopathological examination 9 types of benign lesions in 7 participants were detected and included: 1 case of hamartoma, seborrhoeic wart, hemorrhagic lesion, obliterative bronchiolitis, nodular goiter, tubular bronchial

Table II. Structure of males based on location of pulmonary nodules

\begin{tabular}{lcc}
\hline \multirow{2}{*}{ LUNG } & \multicolumn{2}{c}{$\mathbf{N}=35$} \\
\cline { 2 - 3 } & $\mathbf{N}$ & $\%$ \\
\hline Right & 20 & 57.1 \\
\hline Left & 13 & 37.1 \\
\hline Both & 2 & 5.7 \\
\hline LoBE (SEGMENTS) & \multicolumn{3}{c}{$\mathbf{N}=35$} \\
\cline { 2 - 3 } & $\mathbf{N}$ & $\%$ \\
\hline Upper (1-3) & 18 & 51.4 \\
\hline Lower (6-10) & 13 & 37.1 \\
\hline Middle and uvula (4-5) & 3 & 8.6 \\
\hline Upper and lower & 1 & 8.3 \\
\hline
\end{tabular}

Table III. Histopathological type of lung cancer detected in 7 subjects

\begin{tabular}{llc}
\hline HistoPATHOLOGICAL TYPE & \multicolumn{2}{c}{$\mathbf{N}=7$} \\
\cline { 2 - 3 } OF LUNG CANCER & $\mathbf{N}$ & $\%$ \\
\hline Adenosquamous carcinoma & 2 & 28.6 \\
\hline Adenocarcinoma: & 5 & 71.4 \\
\hline Papillary type of adenocarcinoma & 1 & 14.3 \\
\hline Acinar type of adenocarcinoma & 1 & 14.3 \\
\hline Solid (NOS) type of adenocarcinoma & 3 & 42.8 \\
\hline
\end{tabular}

gland adenoma and hyperplastic bronchial polyp, and 2 cases of silicosis. Lung cancer was diagnosed by means of invasive procedures in 7 (21.9\%) of the 32 participants with 7 potentially malignant pulmonary nodules. Within 5 males adenocarcinoma type was proved in 5 potentially malignant pulmonary nodules: 1 case presented papillary adenocarcinoma, 1 case acinar adenocarcinoma and 3 cases belonged to solid [NOS (not otherwise specified)] type of adenocarcinoma. In 2 males with 2 type $C$ pulmonary nodules adenosquamous carcinomas were histologically proved (Table III). Six out of 7 cases $(85.7 \%)$ were classified as pathological stage I, 1 case out of 7 (14.3\%) presented pathological stage IV.

Specificity for the baseline screening protocol was 96.4\%. Sensitivity in baseline screening was not obtained, because the study was still in the follow-up phase. There was no significant difference in mean age among males with lung cancer and without lung cancer. There was also no significant difference in years of smoking duration, number of cigarettes smoked per day, pack-years and number of pulmonary nodules among men with lung cancer and without lung cancer. There was no significant correlation between positive family cancer history, current smoking, exposure to asbestos and prevalence of lung cancer. However, these results could be due to the very small number of studied participants.

\section{Discussion}

Since the National Lung Screening Trial (NLST) proved a $20.1 \%$ reduction in lung cancer mortality rate in participants screened by low dose chest CT in comparison to chest radiograph, low dose CT (LDCT) became a major technique used in lung cancer screening studies [8]. Moreover, computer aided diagnosis (CAD) systems in chest CT improved accuracy in detecting pulmonary nodules considered as potentially early stages of lung cancer $[2,3,5,7]$. Detecting lung cancer at an early stage improved treatment and the survival rate. The aim of lung cancer screening trials still remained detection of pulmonary nodules and distinguishing benign from malignant ones based on their morphological characteristics, to decrease the lung cancer mortality rate on one hand and diminish over-diagnosis and un- 


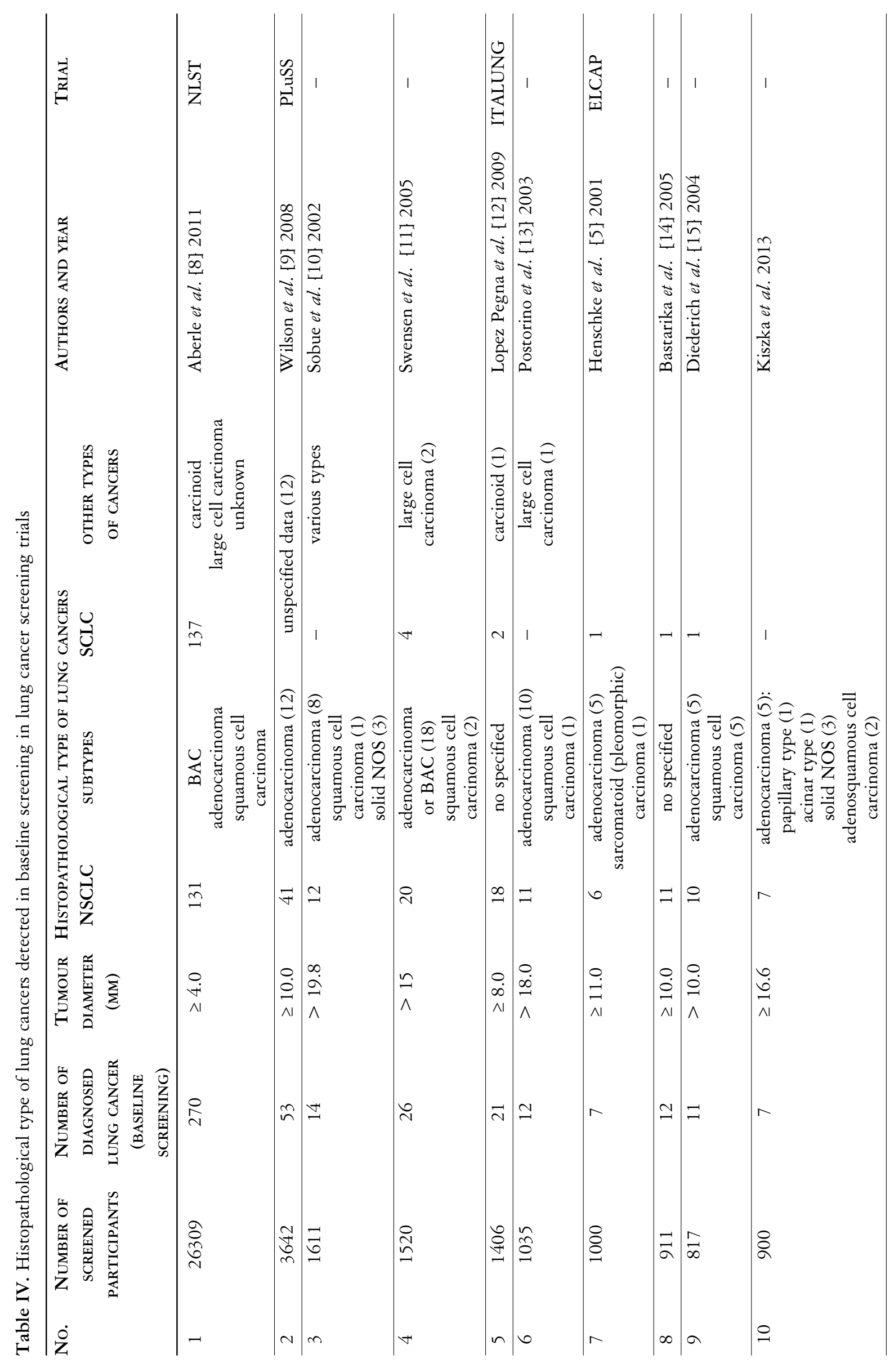




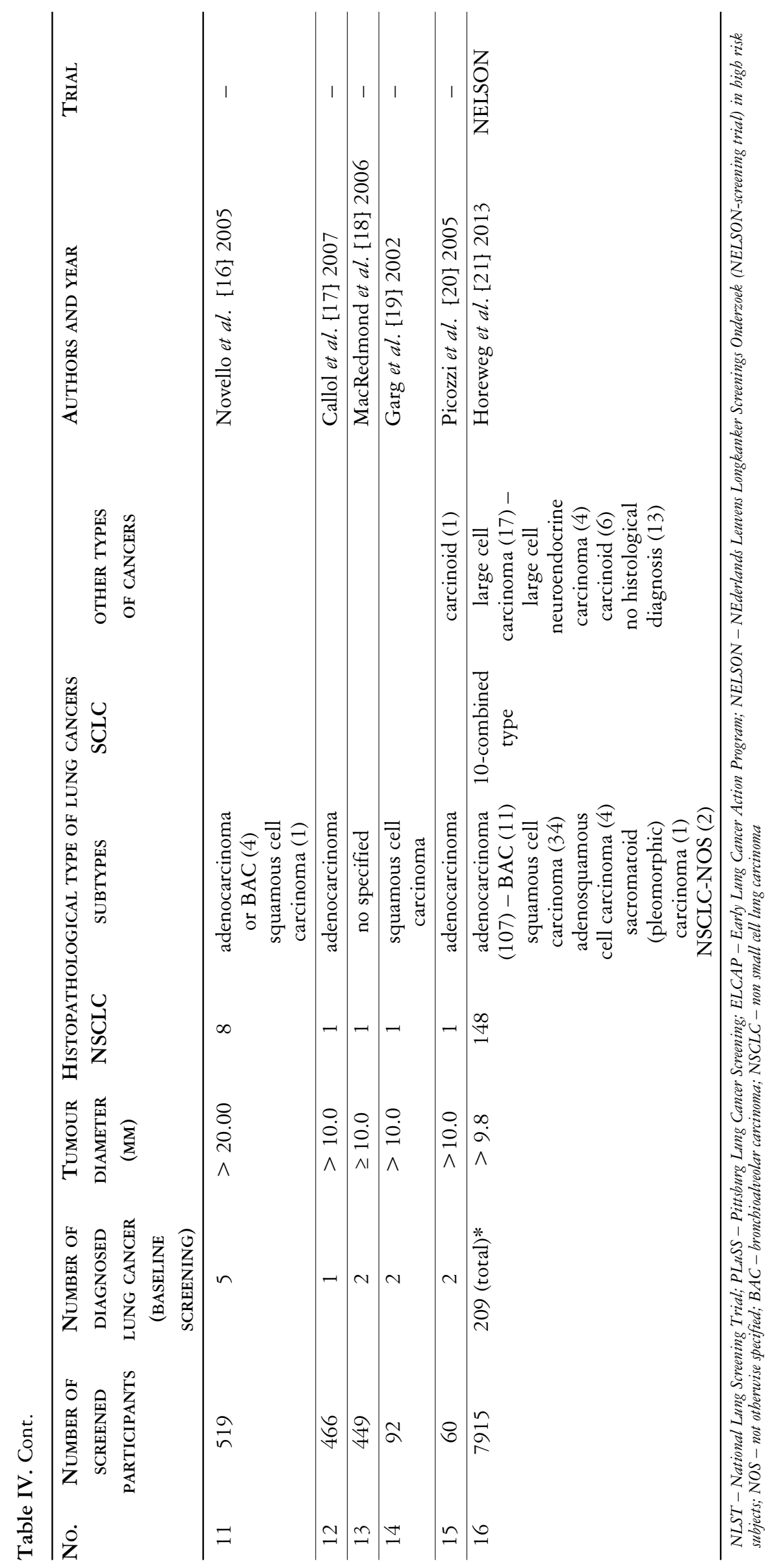


necessary invasive or surgical procedures on the other hand [7-9]. However, it was always histopathological examination which established the final diagnosis.

In this study we revealed solid pulmonary nodules in $92 \%(\mathrm{n}=831)$ of participants in baseline screening, which was much higher than that reported in the literature $(5-51 \%)$ [7-20]. We obtained specificity of $96.4 \%$ of baseline screening, which was similar to the published experience (92-99.6\%) [7-20]. Sensitivity remains unknown, for our study is still ongoing. We proved lung cancer nodules in $0.7 \%(\mathrm{n}=7)$ of subjects $(0.2-3.7 \%$ in other studies), potentially malignant non-lung cancer nodules in $3.5 \%(\mathrm{n}=32)$ of cases (1-49\% reported in the literature) and benign nodules in $95.6 \%(\mathrm{n}=861)$ of males, which was similar to the other studies (77-100\%) [22]. We classified all male smokers to group A, B or C according to NELSON criteria, which referred to nodule volumetry $[3,4]$. Similarly to other trials, an annual scan for small nodules $(\varnothing \leq 5 \mathrm{~mm})$ was performed, due to their very low likelihood of malignancy $[1,5,6]$. The main differences between the protocol and other trials occurred within group B (indeterminate nodules), with larger cutoff of diameter and volumetry in our study $(8-10 \mathrm{~mm}$, $50-500 \mathrm{~mm}^{3}$ in comparison with $5-8 \mathrm{~mm} ; 35-135 \mathrm{~mm}^{3}$ in other trials) [22]. It was in order to avoid over-diagnosis and unnecessary surgical procedures of indeterminate nodules in potentially healthy male smokers, rather with the focus on delaying the decision of invasive diagnostics, unless nodule growth was proved $[1,4,5]$. However, considering the low lung cancer detection rate in the studied sample $(0.7 \%)$, which remained much lower than the prevalence of potentially malignant nodules (4.3\%), and considering the large total number of nodules detected in male smokers (92\%) in studied participants in Cracow, the question about changing (decreasing) the cut-off size of indeterminate nodules for the Polish population remained open.

Based on sub-type of histological analyses, adenocarcinoma type (with papillary, acinar, and solid [NOS] variants) and adenosquamous cell carcinoma type within potentially malignant pulmonary nodules in the studied sample of elderly male smokers in Cracow were proved.

NSCLC (non-small cell lung carcinoma) is the most often proved lung cancer type in the literature (Table IV). Among this group adenocarcinoma with BAC (bronchioalveolar type) was detected in over half of cases. The second most often detected lung cancer type was squamous cell carcinoma. Small cell lung carcinomas (SCLC), large cell carcinomas, and carcinoid were diagnosed in several single cases (Table IV). In our study $85.7 \%$ of cases were classified as pathological stage I and $14.3 \%$ of cases presented pathological stage IV, in comparison to $70.8 \%$ (stage IA) and $8.1 \%$ (IIIB + IV) reported in Horeweg's paper summarizing the long-term Dutch-Bel- gian part of the NELSON study [21]. Our results, however, remained similar to other trials $[8,12,13]$.

Benign lesions were also histologically proved and presented various types of benign diseases. Median volume of malignant nodules in our study is slightly higher than that reported in the literature, but it could also be caused by a different cut-off of median diameter, which was cleared above $[3,4,7]$. Further studies and follow-up are needed to improve guidelines for management of pulmonary nodules detected on CT scans [6].

\section{Conclusions}

We determined morphological characteristics of a group with potentially malignant pulmonary nodules in 39 high-risk male smokers selected from a studied population in Cracow (Poland). We estimated median volume for each type $\mathrm{C}$ pulmonary nodule and for each type $\mathrm{A}$ and $\mathrm{B}$ pulmonary nodule within group $\mathrm{C}$. The prevalence of location of type $\mathrm{C}$ nodules in the right lung and in the upper lobe was observed. In 7 cases lung cancer was histologically proved with 5 cases of adenocarcinoma type and 2 cases of adenosquamous carcinomas. In 7 subjects 9 nodules were found to be benign lesions.

The project was funded by the Ministry of Science and Higher Education, 331/N-HOL UMCG/2008/09/0.

The authors declare that there were no study sponsors involved in the study design.

\section{References}

1. van Klaveren RJ, Oudkerk M, Prokop M, et al. Management of lung nodules detected by volume CT scanning. N Engl J Med 2009; 361: 2221-2229.

2. Kiszka K, Haduch J, Pasowicz M. Application of computer aided diagnosis (CAD) in clinical imaging. Presentation of the value and current directions in CAD development in various imaging techniques based on literature review. Przegl Lek 2009; 66: $437-447$

3. Xu DM, Gietema H, de Koning $\mathrm{H}$, et al. Nodule management protocol of the NELSON randomized lung cancer screening trial. Lung Cancer 2006; 54: 177-184.

4. van Iersel CA, de Koning HJ, Draisma G, et al. Risk-based selection from the general population in screening trial: selection criteria, recruitment and power for the Dutch-Belgian randomized lung cancer multi-slice CT screening trial (NELSON). Int J Cancer 2007; 120: 868-874.

5. Zhao Y, de Bock GH, Vliegenthart R, et al. Performance of computer-aided detection of pulmonary nodules in low-dose CT: comparison with double reading by nodule volume. Eur Radiol 2012; 22: 2076-2084.

6. MacMahon H, Austin JH, Gamsu G, et al. Gudelines for management of small pulmonary nodules detected on CT scans: a statement from the Fleischner Society. Radiology 2005; 237 : 395-400

7. Henschke CI, Naidich DP, Yankelevitz DF, et al. Early lung cancer action project: initial findings on repeteat screenings. Cancer 2001; 92: 153-159.

8. Aberle DR, Adams AM, Berg CD, et al. National Lung Cancer Screening Trial Research Team. Reduced lung mortality with 
low-dose computed tomographic screening. N Engl J Med 2011; 365: 395-409.

9. Wilson DO, Weissfeld JL, Fuhrman CR, et al. The Pittsburg Lung Cancer Screening (PLuSS): outcomes within 3 years of a first computed tomography scan. Am J Respir Crit Care Med 2008; 178: 956-961.

10. Sobue T, Moriyama N, Kaneko M, et al. Screening for lung cancer with low-dose helical computed tomography: anti-lung cancer association project. J Clin Oncol 2002; 20: 911-920.

11. Swensen SJ, Jett JR, Hartman TE, et al. Lung cancer screening with CT: Mayo Clinic experience. Radiology 2003; 226: 756-761.

12. Lopes Pegna A, Picozzi G, Mascalchi M, et al. ITALUNG Study Research Group. Design, recruitment and baseline results of the ITALUNG trial for lung cancer screening with low-dose CT. Lung Cancer 2009; 64: 34-40.

13. Pastorino U, Bellomi M, Landoni C, et al. Early lung cancer detection with spiral CT and position emission tomography in heavy smokers:2-year results. Lancet 2003; 362: 593-597.

14. Bastarrika G, García-Velloso MJ, Lozano MD, et al. Early lung cancer detection using spiral computed tomography and position emission tomography. Am J Respir Crit Care Med 2005; 171: 1378-1383.

15. Diederich S, Thomas M, Semik M, et al. Screening for early lung cancer with low-dose spiral computed tomography: results of annual follow-up examinations in asymptomatic smokers. Eur Radiol 2004; 14: 691-702.

16. Novello S, Fava C, Borasio P, et al. Three-year findings of an early lung cancer detection feasibility study with low-dose spiral computed tomography in heavy smokers. Ann Oncol 2005; 16: 1661-1666

17. Callol L, Roig F, Cuevas A, et al. Low-dose CT:a useful and accessible tool for the early diagnosis of lung cancer in selected populations. Lung Cancer 2007; 56: 217-221.

18. MacRedmond R, McVey G, Lee M, et al. Screening for lung cancer using low dose CT scanning: results of 2 year follow up. Tho$\operatorname{rax} 2006$; 61: 54-56.

19. Garg K, Keith RL, Byers T, et al. Randomized controlled trial with low-dose spiral CT for lung cancer screening: feasibility study and preliminary results. Radiology 2002; 225: 506-510.

20. Picozzi G, Paci E, Lopez Pegna A, et al. Screening of lung cancer with low dose spiral CT: results of a three year pilot study and design of the randomized controlled trial "Italung-CT". Radiol Med 2005; 109: 17-26.

21. Horeweg N, van der Aalst CM, Thunnissen E, et al. Characteristics of lung cancers detected by computer tomography screening in the randomized NELSON trial. Am J Respir Crit Care Med 2013; 187: 848-854.

22. Bach PB, Mirkin JN, Oliver TK, et al. Benefits and harm of CT screening for lung cancer. JAMA 2012; 307: 2418-2429.

\section{Address for correspondence}

\section{Kinga Kiszka}

Department of Radiology, John Paul II Hospital

Pradnicka 80

31-202 Kracow, Poland

tel. +48126142526

$\mathrm{fax}+48126142500$

e-mail:k.kiszka@szpitaljp2.krakow.pl,kinga_kiszka@wp.pl 